九州大学学術情報リポジトリ

Kyushu University Institutional Repository

\title{
Clinical and MRI characteristics of acute encephalopathy in congenital adrenal hyperplasia
}

Lee, Sooyoung

Department of Pediatrics, Graduate School of Medical Sciences, Kyushu University

Sanefuji, Masafumi

Department of Pediatrics, Graduate School of Medical Sciences, Kyushu University

Watanabe, Kenj i

Department of Pediatrics, Kagoshima University Graduate School of Medical and Dental Sciences

Uematsu, Ayumi

Department of Endocrinology and Metabolism, Shizuoka Children's Hospital

他

http://hdl. handle. net/2324/25607

出版情報 : Journal of the Neurological Sciences. 306 (1/2)，pp.91-93，2011-07-15. Elsevier バージョン:

権利関係: (C) 2011 Elsevier B.V. 
Title: Clinical and MRI characteristics of acute encephalopathy in congenital adrenal hyperplasia

Authors: Sooyoung Lee ${ }^{1}$, Masafumi Sanefuji ${ }^{1}$, Kenji Watanabe ${ }^{2}$, Ayumi Uematsu ${ }^{3}$, Hiroyuki Torisu ${ }^{1}$, Haruhisa Baba ${ }^{1}$, Ryutaro Kira ${ }^{1}$, Yui Takada ${ }^{1}$, Yoshito Ishizaki ${ }^{1}$, Mitsuo Toyoshima ${ }^{2}$, Fumio Aragaki ${ }^{4}$, Daisuke Hata ${ }^{5}$, and Toshiro Hara ${ }^{1}$

Affiliations: ${ }^{1}$ Department of Pediatrics, Graduate School of Medical Sciences, Kyushu University, Fukuoka, Japan

${ }^{2}$ Department of Pediatrics, Kagoshima University Graduate School of Medical and Dental Sciences, Kagoshima, Japan.

${ }^{3}$ Department of Endocrinology and Metabolism, Shizuoka Children's Hospital, Shizuoka, Japan

${ }^{4}$ Department of Pediatrics, Kurashiki Central Hospital, Kurashiki City, Okayama, Japan

${ }^{5}$ Department of Pediatrics, Kitano Hospital, Osaka, Japan

Correspondence: Masafumi Sanefuji, MD, PhD, Department of Pediatrics, Graduate School of Medical Sciences, Kyushu University, 3-1-1 Maidashi, Higashi-ku, Fukuoka 812-8582, Japan.

TEL: +81-92-642-5421, FAX: +81-92-642-5435

E-mail: sane26@pediatr.med.kyushu-u.ac.jp

Author's keywords: pediatric neurology; acute encephalopathy; brain imaging; cognitive neuroscience 


\section{Declaration of competing interests:}

The authors have no conflicts of interest to declare.

\section{Funding:}

This work was supported in part by a Grant-in-Aid for Scientific Research from the Ministry of Education, Culture, Sports, Science and Technology of Japan. 


\begin{abstract}
Acute encephalopathy in childhood is frequently associated with common infections, especially in East Asia. Various types have been identified although many cases remain unclassified. Congenital adrenal hyperplasia $(\mathrm{CAH})$ is an autosomal recessive disease presenting impairment of cortisol biosynthesis. We report three $\mathrm{CAH}$ children with acute infection-related encephalopathy. They exhibited disturbed consciousness or seizures, which did not improve after glucocorticoid administration, accompanied by clinical and laboratory findings of adrenal insufficiency. Brain MRI disclosed various patterns of white matter lesions, suggesting different types of acute encephalopathy such as clinically mild encephalitis/encephalopathy with a reversible splenial lesion or hemiconvulsion-hemiplegia syndrome. Acute encephalopathy should be considered and brain MRI immediately performed when impairment of consciousness does not improve after intravenous glucocorticoid administration in $\mathrm{CAH}$ patients. Further research is required to elucidate the epidemiology and pathogenic mechanisms of acute encephalopathy in $\mathrm{CAH}$.
\end{abstract}

Key Words: child; congenital adrenal hyperplasia; encephalitis; encephalopathy; MRI; white matter 


\section{Introduction}

Encephalitis is defined as inflammation of the brain, presenting various neurological manifestations such as disturbance of consciousness or seizures. In clinical practice, the diagnosis is based on symptoms, radiological findings and inflammatory markers such as pleocytosis of the cerebrospinal fluid (CSF). The less specific term "encephalopathy" is used when neurological manifestations suggest encephalitis but inflammation is not identified in the brain or CSF [1]. Acute encephalopathy in childhood is frequently associated with common infections, especially in East Asia [2]. Only a fraction of the encephalopathy is accounted for by inherited metabolic disorders such as fatty acid oxidation defects and carnitine palmitoyltransferase deficiency, at least, in Japan [2]. Others are classified into various types including Reye syndrome [3], hemorrhagic shock and encephalopathy [4] or acute necrotizing encephalopathy [5], although many cases remain unclassified. Recently, new types have been identified according to MRI findings, such as clinically mild encephalitis/encephalopathy with a reversible splenial lesion (MERS) [6-7] and acute encephalopathy with biphasic seizures and late reduced diffusion (AESD) [1].

Congenital adrenal hyperplasia $(\mathrm{CAH})$ is an autosomal recessive disease which manifests impairment of cortisol biosynthesis. The impairment results in underproduction of cortisol and aldosterone, and overproduction of adrenocorticotropic hormone (ACTH), corticotrophin, 17-hydroxyprogesterone and androgens. A Japanese nation-wide survey reported on the clinical symptoms of acute encephalopathy in treated children with $\mathrm{CAH}$ [8]. The study demonstrated that CAH children were highly susceptible to infection-associated acute encephalopathy and that acute adrenal insufficiency might relate to the onset. To our best knowledge, there are no reports to 
describe the detailed characteristics of acute encephalopathy in patients with $\mathrm{CAH}$. We report here clinical and MRI findings of three $\mathrm{CAH}$ children presenting with acute encephalopathy.

\section{Case reports}

\section{Patient 1}

A 9-year-old girl was admitted (day 1) because of a disturbance in consciousness after 2 days of fever and vomiting, during which she could not take hydrocortisone. She had been diagnosed as simple virilizing subtype of 21ß-hydroxylase deficiency (21-OHD) while a neonate and took replacement therapy with oral hydrocortisone. She showed lethargy and delirium without other physical and neurological abnormalities. Her state of consciousness was unchanged after intravenous hydrocortisone. An arterial blood gas showed metabolic acidosis: $\mathrm{pH}$ 7.241, pCO2 34.8 $\mathrm{mmHg}$, base deficit $-11.8 \mathrm{mEq} / \mathrm{L}$ and anion gap $10.6 \mathrm{mmol} / \mathrm{L}$. Laboratory data were the following: white blood cell count 10,140/ $\mu \mathrm{L}$; C-reactive protein (CRP) $11.02 \mathrm{mg} / \mathrm{dL}$; glucose $111 \mathrm{mg} / \mathrm{dl}$; Na $125 \mathrm{mEq} / \mathrm{L} ; \mathrm{K} 4.9 \mathrm{mEq} / \mathrm{L}$; aspartate aminotransferase (AST) 96 IU/L; alanine aminotransferase (ALT) 29 IU/L; lactate dehydrogenase (LDH) 618 IU/L; creatinine kinase $7306 \mathrm{U} / \mathrm{L}$; ammonia $30 \mu \mathrm{g} / \mathrm{dL}$; lactate $0.77 \mathrm{mmol} / \mathrm{L}$. Amino acid analysis and acylcarnitine profiles revealed no abnormalities. CSF examination revealed normal cell counts $(4 / \mu \mathrm{l})$ with normal protein level $(11 \mathrm{mg} / \mathrm{dL})$. Influenza A virus was detected by rapid antigen test. Electroencephalography showed generalized bilaterally synchronous slow waves without epileptiform discharges. There were no abnormalities in T1 and T2 weighted, and fluid attenuated inversion recovery (FLAIR) images of MRI. However, diffusion-weighted images (DWI) of MRI revealed high intensity lesions 
diffusely in white matter and in the entire corpus callosum with reduced apparent diffusion coefficients (ADC; Fig. 1A). She was treated with oseltamivir, steroid pulse therapies, intravenous immunoglobulin and therapeutic hypothermia with mechanical ventilation. After the hypothermia, her level of consciousness improved gradually. DWI on day 17 showed complete resolution of the lesions. She showed no neurological sequelae.

\section{Patient 2}

A 1-year 5-month-old boy showed disturbance of consciousness after 1 day of fever and diarrhea. He had been diagnosed as salt-wasting 21-OHD through neonatal screening tests and supplemented with oral hydrocortisone and fluorocortisol, which he could not take at that time. He was admitted as his level of consciousness did not improve after three times his usual dose of oral hydrocortisone. On admission (day1) he was comatose and exhibited complex partial status epilepticus. Electroencephalography showed repetitive spike-and-wave patterns in the left hemisphere, predominantly in the temporal area. Intravenous hydrocortisone was administered but his level of consciousness did not recover. An arterial blood gas showed metabolic acidosis: $\mathrm{pH}$ 7.227, pCO2 45.5 mmHg, base deficit $-9.0 \mathrm{mEq} / \mathrm{L}$ and anion gap $19.7 \mathrm{mmol} / \mathrm{L}$. Laboratory data were the following: white blood cell count 9,400/ $\mu \mathrm{L}$; CRP $0.4 \mathrm{mg} / \mathrm{dL}$; glucose 38 mg/dl; Na 134 mEq/L; K 5.4 mEq/L; AST 266 IU/L; ALT 78 IU/L; LDH 732 IU/L; ACTH 1430 pg/ml [reference range: 7.4-55.7]; ammonia $61 \mu \mathrm{g} / \mathrm{dL}$. Blood lactate level was modelately high $(3.94 \mathrm{mmol} / \mathrm{L})$ on admission but normalized soon. CSF examination revealed mild pleocytosis $(13 / \mu \mathrm{l})$ with normal protein level $(20 \mathrm{mg} / \mathrm{dL})$. No specific infectious agents were identified by extensive work-up. FLAIR images of 
MRI disclosed multiple high intensity lesions in the cerebral white matter (Fig. 1B). He was treated with intravenous administration of hydrocortisone, barbiturate and immunoglobulin with mechanical ventilation. FLAIR images of MRI on day 16 showed partial resolution of the high intensity lesions. He showed no neurological sequelae except for epilepsy.

\section{Patient 3}

A 2-year-11-month-old boy was admitted (day 1) because of repeated, prolonged seizures after 1 day of vomiting, diarrhea and fever. He received twice his usual dose of hydrocortisone. He had been diagnosed as salt-wasting 21-OHD through neonatal screening tests and supplemented with oral hydrocortisone and fluorocortisol. He had exhibited mild developmental retardation. He was semicomatose and had clusters of generalized seizures. An arterial blood gas showed metabolic acidosis: $\mathrm{pH}$ 7.12, pCO2 $38.0 \mathrm{mmHg}$, base deficit $-16.1 \mathrm{mEq} / \mathrm{L}$ and anion gap $23.7 \mathrm{mmol} / \mathrm{L}$. Laboratory data were the following: white blood cell count $12,560 / \mu \mathrm{L} ; \mathrm{CRP} 6.7 \mathrm{mg} / \mathrm{dL}$; glucose 54 mg/dl; Na 130 mEq/L; K 4.2 mEq/L; AST 52 IU/L; ALT 21 IU/L; LDH 300 IU/L; ACTH 146 pg/ml; ammonia 89 g/dL; Blood lactate level was moderately high (3.51 mmol/L) but normalized soon. CSF examination revealed normal cell counts $(4 / \mu \mathrm{l})$ with normal protein level $(25 \mathrm{mg} / \mathrm{dL})$. No specific infectious agents were identified by extensive work-up. He was treated with intravenous administration of diazepam and hydrocortisone. His fever resolved and he became alert on day 2 of his illness. However, conjugate eye deviation to the right and clusters of left hemiconvulsions appeared on day 4. DWI of MRI revealed high intensity lesions in the right frontal subcortical white matter with reduced ADC (Fig. 1C). He was treated with 
intravenous hydrocortisone, edaravone and hyperbaric oxygen therapy. DWI on day 11 showed high intensity lesions in the right cortical hemisphere with reduced ADC (Fig. 1D). He exhibited mild left hemiparesis and epilepsy as sequelae.

\section{Discussion}

We report three $\mathrm{CAH}$ children with acute infection-associated encephalopathy. They showed impaired consciousness or seizures, which did not improve after intravenous administration of glucocorticoid. At the onset, they exhibited clinical symptoms and laboratory findings of adrenal insufficiency: vomiting, diarrhea, hypoglycemia, hyponatremia, hyperkalemia, metabolic acidosis or elevation of ACTH. Brain MRI demonstrated various patterns of white matter lesions.

Variable clinical and MRI findings in our patients indicate that acute infection-associated encephalopathy in $\mathrm{CAH}$ children may not relate to the specific type of encephalopathy. In patient 1, DWI showed high intensity lesions in extensive white matter areas and the entire corpus callosum, which disappeared at follow-up MRI. This transient MRI abnormality and benign clinical course are consistent with MERS type 2 [6]. In patient 2, MRI showed multiple lesions in the cerebral white matter. We could not classify his type of encephalopathy. In patient 3 , lesions in the right hemisphere and clusters of left hemiconvulsions with subsequent hemiparesis led to the diagnosis of hemiconvulsion-hemiplegia syndrome (HHS) [9]. The serial change in the high intensity lesions from subcortical white matter to cortex conforms well with a previous report [10]. The lesions in the three patients were variable but predominant in white matter. 
The white matter lesions in these cases may reflect white matter fragility in CAH. MRI studies have demonstrated subclinical white matter abnormalities in adult patients with $\mathrm{CAH}$ [11-13]. The distribution of these abnormalities was variable: focal, diffuse and both focal and diffuse. In rat pups, hydrocortisone may promote the differentiation into oligodendrocytes that provide myelin in the white matter [14]. Conversely, glucocorticoid administration inhibits the proliferation of oligodendrocyte progenitors in adult rats [15] and delays myelination of the corpus callosum, a major white matter tract in fetal sheep [16]. Patients with CAH can be overexposed to corticotrophin, 17-hydroxyprogesterone and androgens, and underexposed to cortisol and aldosterone, before and after birth. Additionally, their cortisol levels are dependent on exogenous replacement and different from the natural circadian variation [17]. These imbalances of hormonal factors might disrupt the normal formation and maintenance of white matter [18] and provide the basis of the lesions in CAH children.

The pathogenic mechanism of acute encephalopathy in children with $\mathrm{CAH}$, as well as no underlying disease, remains unknown, although adrenal insufficiency may play a role in its onset, as previously suggested [8]. The white matter lesions in our patients are unlikely to be explained by hypoxic/ischemic or hypoglycemic encephalopathy due to adrenal crisis as reported in a CAH child [19]. In that encephalopathy, MRI usually demonstrates diffuse lesions predominantly in the cortex, thalamus, basal ganglia or hippocampus. In MERS (patient 1) and HHS (patient 3), one of the pathogenic mechanisms is postulated to be inflammation $[7,20]$. In cases of virus infection, corticosteroids are produced in response to pro-inflammatory cytokines and downregulate these entities [21]. Endogenous or supplemented corticosteroids may be too insufficient to regulate these cytokines in some $\mathrm{CAH}$ patients. This corticosteroids 
insufficiency might fail to suppress excessive cytokines and trigger acute encephalopathy. To the best of our knowledge, the association of acute encephalopathy with CAH has not been reported except in Japan [8]. One possible explanation for this and for the high incidence of acute encephalopathy in East Asia is that Asian people might have genetic backgrounds that facilitate the development of encephalopathy [22]. The infection-related acute adrenal insufficiency might induce the onset of acute encephalopathy in $\mathrm{CAH}$ individuals with such a genetic predisposition.

\section{Conclusion}

In $\mathrm{CAH}$ children, various types of acute encephalopathy can develop following common infections. Acute encephalopathy should be considered and brain MRI including DWI immediately performed when impairment of consciousness does not improve after intravenous glucocorticoid administration. Further research is required to elucidate the epidemiology and pathogenic mechanisms of the association.

Acknowledgments: This work was supported in part by Grants-in-Aid for Scientific Research to M.S., H.T. and R.K. from the Ministry of Education, Culture, Sports, Science and Technology of Japan (No. 21790995, No. 20591225 and No. 21591326). 


\section{References}

[1] Takanashi J, Oba H, Barkovich AJ, Tada H, Tanabe Y, Yamanouchi H, et al. Diffusion MRI abnormalities after prolonged febrile seizures with encephalopathy. Neurology 2006;66:1304-9.

[2] Mizuguchi M, Yamanouchi H, Ichiyama T, Shiomi M. Acute encephalopathy associated with influenza and other viral infections. Acta Neurol Scand 2007;Suppl 186:45-56.

[3] Gosalakkal JA, Kamoji V. Reye syndrome and reye-like syndrome. Pediatr Neurol 2008;39:198-200.

[4] Levin M, Hjelm M, Kay JD, Pincott JR, Gould JD, Dinwiddie R, et al. Haemorrhagic shock and encephalopathy: a new syndrome with a high mortality in young children. Lancet 1983;2:64-7.

[5] Mizuguchi M, Abe J, Mikkaichi K, Noma K, Yoshida K, Yamanaka T, et al. Acute necrotizing encephalopathy of childhood: a new syndrome presenting with multifocal, symmetric brain lesions. J Neurol Neurosurg Psychiatry 1995;58:555-61.

[6] Tada H, Takanashi J, Barkovich AJ, Oba H, Maeda M, Tsukahara H, et al. Clinically mild encephalitis/encephalopathy with a reversible splenial lesion. Neurology 2004;63:1854-8.

[7] Takanashi J, Imamura A, Hayakawa F, Terada H. Differences in the time course of splenial and white matter lesions in clinically mild encephalitis/encephalopathy with a reversible splenial lesion (MERS). J Neurol Sci 2010;292:24-7. 
[8] Kawashima Y, Hanaki K, Kinoshita T, Nagaishi J, Kanzaki S. A nation wide survey for the incidence of the central nervous system complications in patients treated for congenital adrenal hyperplasia (in Japanese). Horumon To Rinsho 2002;50:1165-9.

[9] Okumura A, Suzuki M, Kidokoro H, Komatsu M, Shono T, Hayakawa F, et al. The spectrum of acute encephalopathy with reduced diffusion in the unilateral hemisphere. Eur J Paediatr Neurol 2009;13:154-9.

[10] Yoshikawa H, Kitamura T. Serial changes on diffusion-weighted magnetic resonance imaging in encephalitis or encephalopathy. Pediatr Neurol 2006;34:308-11.

[11] Bergamaschi R, Livieri C, Uggetti C, Candeloro E, Egitto MG, Pichiecchio A, et al. Brain white matter impairment in congenital adrenal hyperplasia. Arch Neurol 2006;63:413-6.

[12] Sinforiani E, Livieri C, Mauri M, Bisio P, Sibilla L, Chiesa L, et al. Cognitive and neuroradiological findings in congenital adrenal hyperplasia.

Psychoneuroendocrinology 1994;19:55-64.

[13] Nass R, Heier L, Moshang T, Oberfield S, George A, New MI, et al. Magnetic resonance imaging in the congenital adrenal hyperplasia population: increased frequency of white-matter abnormalities and temporal lobe atrophy. J Child Neurol 1997;12:181-6.

[14] Warringa RA, Hoeben RC, Koper JW, Sykes JE, van Golde LM, Lopes-Cardozo M. Hydrocortisone stimulates the development of oligodendrocytes in primary glial cultures and affects glucose metabolism and lipid synthesis in these cultures. Brain Res 1987;431:79-86 
[15] Alonso G. Prolonged corticosterone treatment of adult rats inhibits the proliferation of oligodendrocyte progenitors present throughout white and gray matter regions of the brain. Glia 2000;31:219-31.

[16] Huang WL, Harper CG, Evans SF, Newnham JP, Dunlop SA. Repeated prenatal corticosteroid administration delays myelination of the corpus callosum in fetal sheep. Int J Dev Neurosci 2001;19:415-25.

[17] Charmandari E, Johnston A, Brook CG, Hindmarsh PC. Bioavailability of oral hydrocortisone in patients with congenital adrenal hyperplasia due to 21-hydroxylase deficiency. J Endocrinol 2001;169:65-70.

[18] Gago N, Akwa Y, Sananes N, Guennoun R, Baulieu EE, El-Etr M, et al. Progesterone and the oligodendroglial lineage: stage-dependent biosynthesis and metabolism. Glia 2001;36:295-308.

[19] Saito Y, Ogawa T, Nagaishi J, Inoue T, Maegaki Y, Ohno K. Laminar cortical necrosis in adrenal crisis: sequential changes on MRI. Brain Dev 2008;30:77-81.

[20] Ichiyama T, Suenaga N, Kajimoto M, Tohyama J, Isumi H, Kubota M, et al. Serum and CSF levels of cytokines in acute encephalopathy following prolonged febrile seizures. Brain Dev 2008;30:47-52.

[21] Padgett DA, Loria RM, Sheridan JF. Steroid hormone regulation of antiviral immunity. Ann N Y Acad Sci 2000;917:935-43.

[22] Morishima T, Togashi T, Yokota S, Okuno Y, Miyazaki C, Tashiro M, et al. Encephalitis and encephalopathy associated with an influenza epidemic in Japan. Clin Infect Dis 2002;35:512-7. 


\section{Figure Legends}

\section{Figure}

MRI findings of the three patients. A: (patient 1) DWI on day 2 demonstrates symmetrical high intensity lesions in extensive white matter areas and the entire corpus callosum. B: (patient 2) FLAIR shows multiple lesions predominantly in the left white matter. C, D: (patient 3) DWI reveals high intensity lesions in the frontal-dominant right hemisphere, predominantly in subcortical white matter on day $4(\mathbf{C})$ and in the cortices on day 11 (D). 
Figure.1
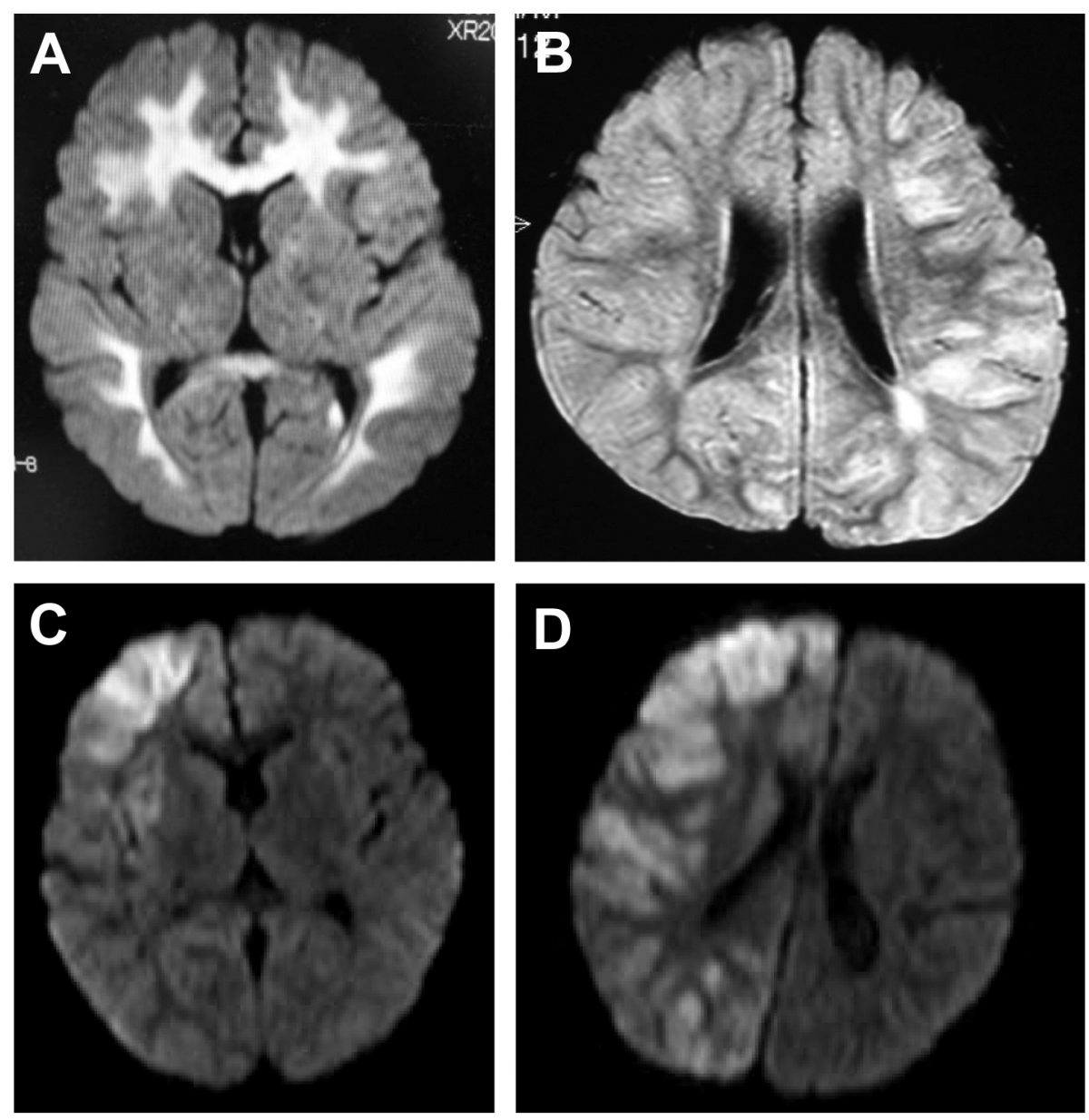